\title{
Reaction Kinetics and Simulations of Ring-Opening Polymerization for the Synthesis of Polybutylene Terephthalate
}

\author{
Jan-Georg Rosenboom ${ }^{1}$, Laura De Lorenzi ${ }^{1}$, Giuseppe Storti ${ }^{1}$, Massimo Morbidelli ${ }^{1, *}$ \\ ${ }^{1}$ ETH Zurich, Institute of Chemical and Bioengineering, Vladimir-Prelog-Weg 1-5/10, \\ 8093 Zurich, Switzerland \\ *massimo.morbidelli@chem.ethz.ch
}

\begin{abstract}
Cyclic polybutylene terephthalate (PBT) was polymerized with 2-ethylhexanoic acid tin(II) salt activated by 1-dodecanol in order to study the reaction mechanisms dominant in ring-opening polymerization (ROP) for polyester synthesis. Initiator-to-monomer content and temperatures were varied from $0.05 \%$ to $0.5 \%$ and $190^{\circ} \mathrm{C}$ to $250^{\circ} \mathrm{C}$, respectively. The living-like character of ROP was confirmed by the characteristic effect of initiator content on cyclic oligomer conversion, along with the linear dependence of the number-average molecular weight upon conversion. The molecular weight distribution is mainly a function of the interplay between chain transfer and transesterification reactions. Since the PBT macrocycles appear in different sizes from 2 to 7 repeat units, each carbonyl group theoretically contributes to ring reactivity, where in this system both size-dependent and size-independent approaches to simulate propagation deliver appreciable results. The corresponding rate constants have been determined and, in contrast to other polymer systems, the proximity of these values to those of chain transfer and transesterification is significant.
\end{abstract}




\section{INTRODUCTION}

Polyester materials such as polyethylene and polybutylene terephthalate (PET and PBT, respectively) rank among the most relevant commercial plastics due to their excellent thermoplastic, crystalline and mechanical properties. They find applications in a wide range of markets from automotive to packaging to textile to medical, with market volumes over $60 \mathrm{Mt}$ $\mathrm{a}^{-1}$ for PET and $1 \mathrm{Mt} \mathrm{a}^{-1}$ for PBT[1-3]. Along the shift towards more sustainability and growing corporate interest in "green” materials that can be made from 100\% renewable resources, also their furan-based counterparts, such as polyethylene and polybutylene furanoate (PEF and PBF, respectively), as well as bio-PET and bio-PBT, are receiving significant attention recently[48].

Traditionally, these polyesters have been produced from synthesis via polycondensation of the diacid and a glycol, e.g. terephthalic acid (TA) and butylene diol (BD) in the case of PBT. In order to reach the high molecular weight polymer required for commercial applications, the removal of the condensation byproduct (e.g. water or an alcohol) is essential. However, volatilization of these compounds becomes increasingly difficult due to the significantly high viscosity and associated diffusion limitations, as the reacting polymer is usually in solid state. As a consequence, the required high vacuum is usually applied over reaction times even on the order of days, which means an energy intensive process, and even degradation and discoloration of the product[1,9-12].

Ring-opening polymerization (ROP) can be the method of choice towards a wide range of polyesters in the future, due to its significant advantages over conventional polycondensation: cyclic oligomers do not feature end-groups and thus render polymerization byproduct removal obsolete, which can reduce reaction time from days down to less than an hour. This allows the adoption of milder operation conditions, such as atmospheric pressure and short exposure to the high processing temperatures. Another advantage is the ability to run termination-free 
"living”-like polymerizations, which enable the controlled synthesis of high molecular-weight polymers through defined initiator amounts[13]. Examples of other polymers commonly produced through ROP are polylactic acid (PLA)[14,15], poly- $\varepsilon$-caprolactone[16,17], polyoxazolines[18,19] and polysiloxanes[20]. In conventional ROPs of the latter, the relief of intramolecular crowding (i.e. ring strain), which results in a negative change in enthalpy, is the driving force of the reaction.

For larger polyesters such as polybutylene terephthalate, the cyclic form is called "macrocycle”, due to the large size of the rings resulting in almost absent ring strain. The polymerization of macrocyclic oligomers is therefore not enthalpically but entropically driven[21]. In other words, according to:

$$
\Delta G=\Delta H-T \cdot \Delta S
$$

the polymerization of macrocycles is almost thermoneutral, i.e. $\Delta H \cong 0$, and the reaction proceeds spontaneously $(\Delta G<0)$ when associated with a positive change in entropy $(\Delta S>0)$. Normally an initiator, such as a metal complex is required to run the reaction. Once the initiator turns active (activation), it opens the cyclic oligomer while becoming part of the linear chain and initiating its growth. In the propagation step, a growing polymer chain reacts with further cyclic oligomers encompassing them into the chain. Tin(II) bis-(2-ethylhexanoate), also referred to as stannous octoate $\mathrm{Sn}(\mathrm{Oct})_{2}$, is probably the most popular initiator regarding ROP of aliphatic esters due to its application in the commercial production of polylactic acid (PLA)[14]. Sn(Oct) 2 has already been accepted by the US Food and Drug Agency (FDA) for application to plastic bottle materials, which could facilitate an easier market entry for food packaging made from polyesters based on $\mathrm{Sn}(\mathrm{Oct})_{2}$-ROP. It has shown good performance and versatility in other studies, and it is soluble in common organic solvents. The polymerization typically involves electrophilic activation of the cyclic oligomer via coordination of the 
exocyclic oxygen to the metal of the activated initiator. Acyl-oxygen bond cleavage and insertion of the cyclics to the metal-oxygen bond lead to ring-opening and chain propagation. Important side reactions that influence the molecular weight distribution rather than oligomer depletion are chain transfer and inter- and intramolecular transesterification reactions[14,15,22,23].

To make a process based on ROP techno-economically viable, the understanding of the governing reaction mechanisms is essential. To this end, computational kinetic models are required to apply the chemical theory and predict the reaction behavior in order to optimize operation conditions and design the appropriate process steps. Chen et al. (2012) have reported on the modelling of fixed-concentration $\mathrm{BuSnClOH}_{2}$-based ROP for PBT and compared their results with conversion and relative molecular weights derived from rheology experiments, considering initiation and propagation as their underlying mechanisms[24]. However, a kinetic model considering chain transfer and transesterification for polyesters such as PET, PBT, PEF and PBF, validated against absolute molecular weight data, has not been published yet. These mechanisms are responsible for the molecular weights and dispersities typically obtained by $\mathrm{ROP}[13]$.

This work focusses on the development of a kinetic model for Sn(Oct) $)_{2}$-based ROP of PBT, in order to understand the governing reaction mechanisms and to describe the experimentally obtained kinetics in terms of conversion $X$ and average properties of molecular weight, typically $M_{n}$ and $M_{w}$. 


\section{MATERIALS AND METHODS}

\section{Materials}

Cyclic PBT oligomers CBT 500 (cyOBT, 98\%) were kindly donated by IQ Tec Germany GmbH (former Cyclics Corporation), 2-ethylhexanoic acid tin(II) (tin octoate, SnOct2, Sigma Aldrich, 95\%) and 1-dodecanol (Acros Organics, 98\%), trifluoroacetic acid (TFA, Fluorochem, 99\%) and potassium trifluoroacetate (K-TFAc, Aldrich, 98\%), tetraethylene glycol dimethyl ether (tetraglyme, Sigma Aldrich, 99\%), hexafluoroisopropanol (HFIP, Fluorochem, 99.9\%) were used as received. The initiators, 1-dodecanol and tetraglyme with molecular sieves were stored in a glove box under nitrogen atmosphere.

\section{Analytics}

${ }^{1} \mathrm{H}$ NMR (300 MHz, 400MHz) spectra were recorded on Bruker Avance III spectrometers. ${ }^{1} \mathrm{H}$ NMR spectra were referenced against the residual solvent signal. Conversion, number- and weight-average absolute molecular weights, $M_{n}$ and $M_{w}$ values of PET and PEF samples were determined by size exclusion chromatography (SEC). An Agilent 1100 GPC/SEC unit was used equipped with two PFG linear M columns (PSS) connected in series with an Agilent 1100 VWD/UV detector operated at $290 \mathrm{~nm}$, a DAWN HELEOS II multi-angle laser light scattering (MALS) detector (Wyatt Technology Europe) followed by an Optilab T-rEX RI detector from Wyatt. Samples were eluted in HFIP with $0.02 \mathrm{M} \mathrm{K-TFAc} \mathrm{at} 1 \mathrm{~mL} \mathrm{~min}^{-1}$ at room temperature. Conversion was evaluated with PSS WinGPC Unichrom software as the fraction of PEF versus total UV signal area. Absolute molecular weights were evaluated with Wyatt ASTRA software and dn/dc values based on our analytical setup $\left(d n / d c(P B T)=0.246 \mathrm{~mL} \mathrm{~g}^{-1}\right)$. 


\section{Synthesis of Polybutylene Terephthalate (PBT) via ROP.}

For every batch of ROP in melt, about $1 \mathrm{~g}$ cyOEF were weighed into a $5 \mathrm{~mL}$ Schlenk tube reactor equipped with a magnetic stirrer in the glove box. The tube was transferred to a heating block and dried at $100^{\circ} \mathrm{C}$ under vacuum for about $30 \mathrm{~min}$. The reactor was removed from the heater and the vacuum was released with nitrogen. The temperature was set to the desired value, and the Schlenk tube was reintroduced to the heating block. After cyOBT had molten after about $5 \mathrm{~min}$, the initiator solution was injected neat or in concentrations of 0.01 to $0.03 \mathrm{M}$ in tetraglyme. Samples were taken over the course of the increasingly viscous reaction mixture using spatulas that were dried in the oven at $120^{\circ} \mathrm{C}$. After the desired reaction time, the product was quenched by immersion in ice water followed by dissolution in pure HFIP and precipitation in THF. The product was collected either by filtration or by centrifuging the mixture and decanting off the solvent. The solid was dried under vacuum at $80^{\circ} \mathrm{C}$, yielding a white powder. ${ }^{1} \mathrm{H}$ NMR (400 MHz, $25^{\circ} \mathrm{C}$, TFA-d) $\delta(\mathrm{ppm})=7.45$ (s, 2H, $-\mathrm{CH}_{\mathrm{ar}}$ ), 4.88 (s, 4H, -CH $\left.\mathbf{C H}_{2}-\mathrm{CH}_{2}-\mathrm{O}\right)$.

\section{RESULTS AND DISCUSSION}

\section{Cyclic Oligomer Characterization and Experimental ROP}

Cyclic PBT oligomers (cyOBT) were available at a constant quality with a melting point measured by DSC at $T_{M}=140^{\circ} \mathrm{C}$. The ring size distribution was revealed by SEC traces that were matched with the (non-quantitative) characterization of MALDI-TOF, as shown in Figure 1a and $\mathbf{b}$. The resulting weight fractions from the SEC (UV) area were: $33 \% C_{2}, 34 \% C_{3}, 16 \%$ $C_{4}, 11 \% C_{5}, 1 \% C_{6}, 5 \% C_{7}$. The cyclic dimer $C_{2}$ and trimer $C_{3}$ were dominant, with larger ring sizes up to $C_{7}$ in lower amounts. This composition was confirmed by HPLC-MS. Note that high oligomer resolution was obtained only using SEC in $15 \% \mathrm{HFIP} / \mathrm{CHCl}_{3}$, which allows for the use of Agilent OligoPore columns with more efficient peak separation than when using pure 
HFIP and compatible columns (shown in Figure 1c). Most of the molecular weights presented in this study were obtained by SEC in pure HFIP due to improved solubility of higher molecular weight polymer. However, the results for cyclic composition and conversion, evaluated as the SEC area ratio of polymer peak versus cyclic oligomer peaks, were found unaffected by the different analytical setup.
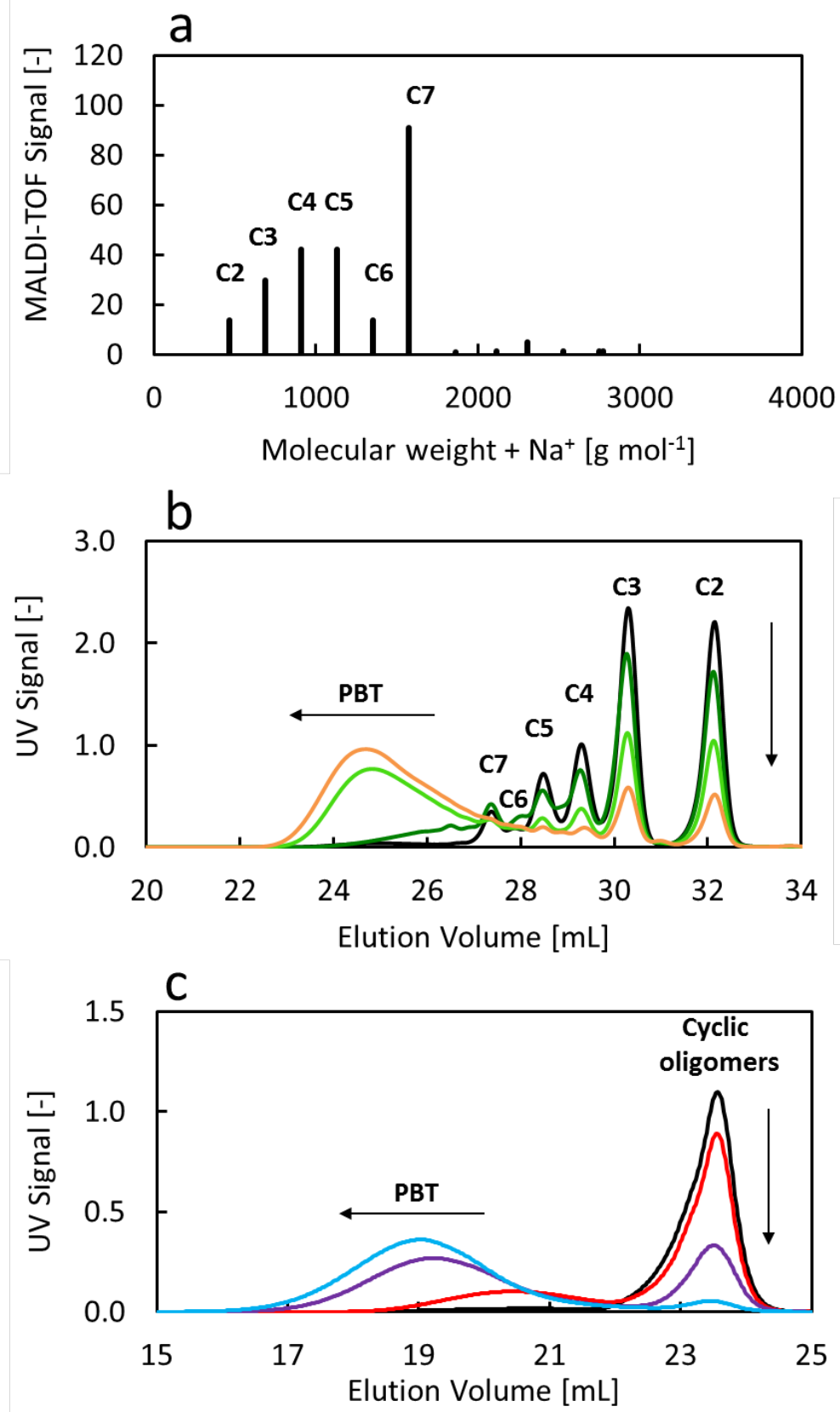

Figure 1. Identification of the cyclic PBT ring size distribution measured by MALDI-TOF (a) and SEC (b) analysis, as well as exemplary visualization of cyclic oligomer conversion to PBT 
polymer along Sn(Oct)2-initiated ROP analyzed over $1 \mathrm{~h}$ with high resolution SEC columns in 15\% HFIP/CHCl 3 (b) eluent and low resolution SEC columns using pure HFIP (c). Arrows show the evolution of monomer and polymer peaks during ROP.

The cyclic raw material was subjected to catalytic ROP initiated by different amounts of initiator at $230^{\circ} \mathrm{C}$. This temperature was chosen since it is above the melting points of both cyOBT $\left(140^{\circ} \mathrm{C}\right)$ and $\mathrm{PBT}\left(223^{\circ} \mathrm{C}\right)$, and resulted in a homogeneous liquid melt along the ROP reaction. Figure 1c shows a typical SEC analysis of ROP using $0.1 \% \mathrm{Sn}(\mathrm{Oct})_{2}$ initiator at $230^{\circ} \mathrm{C}$, which visualizes the consumption of cyOBT by decreasing monomer peaks and the formation of PBT at increasing molecular weight represented by the growing polymer peak. At extended reaction times, the reaction usually equilibrates with a residual cyclics peak of $2 \%$ SEC area (i.e. final conversion of $X=98 \%$ ). Conversion was evaluated as the fraction of polymer peak area relative to total SEC area. Since such areas are proportional to the corresponding mass of repeat units, the conversion is defined as usual:

$$
X=\frac{M_{\text {in }}-M}{M_{\text {in }}},
$$

where $M$ and $M_{i n}$ are the total concentrations of repeat units incorporated in the cyclic oligomers during and at the beginning of the reaction, respectively. In the model, $M$ is calculated as the overall mass of cyclic oligomers divided by the molecular weight of the repeating unit, $m_{r u}$ (= $\left.220.2 \mathrm{~g} \mathrm{~mol}^{-1}\right)$.

The main data set derived and used in this study is presented in Table $\mathbf{1}$ and comprises results in terms of conversion $(X)$, number- and weight-average molecular weights $\left(M_{n}\right.$ and $M_{w}$, respectively) and dispersity $(\bigoplus)$, based on ROPs at $230^{\circ} \mathrm{C}$ using different initiator levels from $0.05 \%$ to $0.5 \%$, expressed as the molar ratio of $\mathrm{Sn}(\mathrm{Oct})_{2}$ and cyclic oligomer repeat units, and a constant ratio of 1-dodecanol $\left(\mathrm{ROH}\right.$, as activator, as discussed later) to $\mathrm{Sn}(\mathrm{Oct})_{2}$ of 15:1. Results below $20 \%$ of conversion were omitted due to significant bias from overlapping 
monomer and polymer peaks in SEC traces. As expected, increased initiator amounts lead to faster conversion and lower molecular weights: additional $\mathrm{Sn}(\mathrm{Oct})_{2}$ results in more active chains, which increases cyclic oligomer consumption per time. The decrease of average molecular weight is mainly a consequence of the increased content of 1-dodecanol, resulting in a large number of dormant chains through chain transfer reactions, thus limiting the average chain length of the final polymer. Dispersity grows very early on from about 1.6 to 2 along the reaction, confirming that transesterification reactions play a very significant role, as will be discussed with more detail in the following.

Table 1. Selected results of experimental ROP for the synthesis of PBT from cyclic oligomers. $X$ is conversion as SEC area fraction of PEF vs. total area. $M_{n}$ and $M_{w}$ are number- and weightaverage molecular weights from SEC-MALS. Activator concentration relative to initiator $D_{\text {in }} / I_{\text {in }}$ was 15 in all cases.

\begin{tabular}{llrrrrrr} 
Entry & Initiator & \multicolumn{1}{c}{ Time } & $\boldsymbol{X}$ & $\boldsymbol{M}_{\boldsymbol{n}}$ & $\boldsymbol{M}_{\boldsymbol{w}}$ & $\boldsymbol{D}$ \\
& & {$\left[{ }^{\circ} \mathrm{C}\right]$} & {$[\mathrm{min}]$} & {$[\%]$} & {$\left[\mathrm{kg} \mathrm{mol}^{-1}\right]$} & {$\left[\mathrm{kg} \mathrm{mol}^{-1}\right]$} & {$[-]$} \\
\hline 1 & $0.05 \% \mathrm{Sn}(\mathrm{Oct})_{2}$ & 230 & 180 & 95 & 19.1 & 38.6 & 2.0 \\
2 & $0.07 \% \mathrm{Sn}(\mathrm{Oct})_{2}$ & 230 & 90 & 96 & 15.7 & 30.9 & 2.0 \\
3 & $0.1 \% \mathrm{Sn}(\mathrm{Oct})_{2}$ & 230 & 60 & 97 & 11.9 & 23.3 & 2.0 \\
4 & $0.2 \% \mathrm{Sn}(\mathrm{Oct})_{2}$ & 230 & 45 & 97 & 8.2 & 15.4 & 1.9 \\
5 & $0.3 \% \mathrm{Sn}(\mathrm{Oct})_{2}$ & 230 & 30 & 98 & 6.7 & 12.7 & 1.9 \\
6 & $0.5 \% \mathrm{Sn}(\mathrm{Oct})_{2}$ & 230 & 15 & 97 & 3.2 & 6.5 & 2.0 \\
\hline 7 & $0.2 \% \mathrm{Sn}(\mathrm{Oct})_{2}$ & 250 & 20 & 98.4 & 8.8 & 17.0 & 1.9 \\
8 & $0.2 \% \mathrm{Sn}(\mathrm{Oct})_{2}$ & 220 & 60 & 97.8 & 9.1 & 16.4 & 1.8 \\
\hline 9 & $0.2 \% \mathrm{Sn}(\mathrm{Oct})_{2}$ & 190 & 90 & 98.5 & 8.8 & 16.6 & 1.9 \\
\hline
\end{tabular}




\section{Mechanisms of Sn(Oct)2-based ROP and Model Development}

\section{Initiator Activation}

Prior to polymerization, $\mathrm{Sn}(\mathrm{Oct})_{2}$ has to be activated and converted into the actual initiator, tin alkoxide, since the carboxylates are weaker nucleophiles in comparison to alkoxides. The corresponding reversible reaction can be sketched as:

$$
I+D \underset{k_{A 2}}{\stackrel{k_{A}}{\rightleftarrows}} R+A
$$

where $I$ is $\mathrm{Sn}(\mathrm{Oct})_{2}, D$ is an OH-bearing compound (typically the alcohol initially injected, in our case high boiling 1-dodecanol, or other protic impurities), $R$ is an activated tin alkoxide, and $A$ is the released 2-Ethylhexanoic acid. Note that, as soon as the polymerization starts proceeding, $D$ and $R$ include also dormant and active chains of any length, as explained in the following section. ROP is usually a living-like reaction, i.e. a termination-free reaction constantly consuming monomer by a fixed number of growing chains. In order to derive the concentration of $\mathrm{Sn}(\mathrm{Oct})_{2}$ molecules actively partaking in the reaction, we have to investigate livingness first, which can be evaluated following the progression of the monomer consumption.

Since the raw material appears as a distribution of ring-sizes $C_{j}$ with $j$ repeat units $(j=2$ to $J$, where $J=7$, cf. Figure 1), we already introduced an effective monomer concentration as the sum of all repeat units in the cyclic oligomers, that is:

$$
M=\sum_{n=2}^{J} j C_{j},
$$


The corresponding mass balance in terms of overall concentration of repeat units is:

$$
\frac{d M}{d t}=-k_{P}^{*} R M
$$

where $R$ is the (constant) concentration of growing chains consuming repeat units by propagation and $k_{P} *$ the corresponding rate coefficient. The superscript * is used to specify the effective nature of this rate constant, fully neglecting the type of cyclic oligomer it belongs to. If livingness is present, the integrated form of Eq. 5 combined with the definition of conversion in Eq. 2, plotted against time, should result in a straight line if propagation involving a constant number of active chains is the only reaction consuming monomer:

$$
M_{\text {in }} \frac{d X}{d t}=-k_{P}^{*} R M_{\text {in }}(1-X) \quad \rightarrow \quad Y=-\ln (1-X)=k_{P}^{*} R t
$$

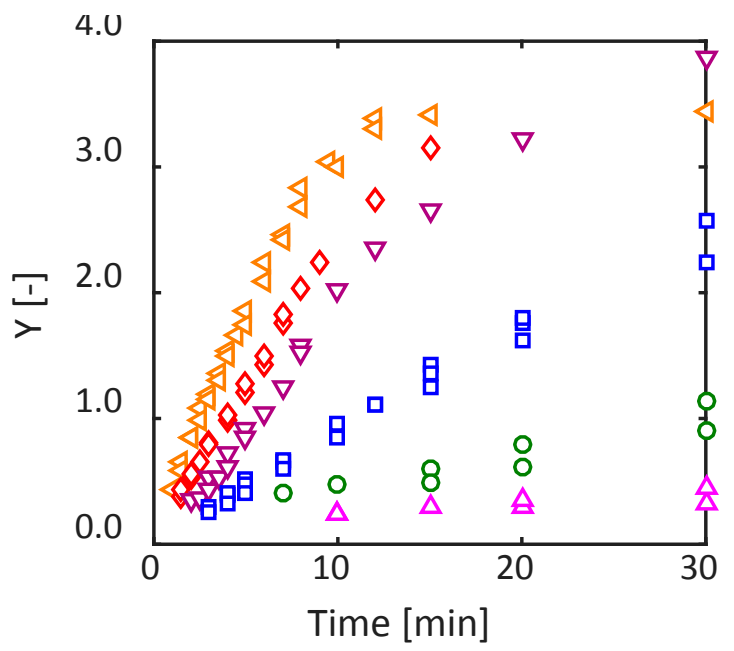

Figure 2. Logarithmic conversion $Y$ over time for ROP of PBT at $230^{\circ} \mathrm{C}$ using molar ratios of initiator $\operatorname{Sn}(\mathrm{Oct})_{2}$ to cyclic monomer repeat units of $0.05 \%(\Delta), 0.07 \%(\circ), 0.1 \%(\square), 0.2 \%(\times)$, $0.3 \%(\diamond)$, and $D_{\text {in }} / I_{\text {in }}$ of $15: 1$. 
Indeed, Figure 2 shows quite linear trends up to about $Y=2.5$ (conversion $X=92 \%$ ), after which deviations become evident and most probably due to the increasing impact of non-ideal effects, such as increased viscosity of the reaction mixture. Thus, equilibrium conditions for the activation reaction can be assumed, and the concentration of $R$ can be evaluated from the mass balance of the different species involved in Eq. 3, as proposed by Yu et al.[14]. Namely, integrating these balances and assuming no initial active species, the actual concentrations of the different species are readily expressed as a function of the initial values (indicated by the subscript “in”) as:

$$
R=I_{\text {in }}-I=D_{\text {in }}-D=A-A_{\text {in }} .
$$

Moreover, the following relationship among the concentrations of the different species (expression of the equilibrium constant of activation) applies:

$$
K_{A}=\frac{R A}{I D} .
$$

Combining Eqs. 7 and 8, the following analytical expression for the residual concentration of $\mathrm{Sn}(\mathrm{Oct})_{2}$ is obtained:

$$
I=\frac{-B+\sqrt{B^{2}+4 I_{i n}\left(K_{A}-1\right)\left(I_{i n}+A_{i n}\right)}}{2\left(K_{A}-1\right)},
$$

where $B=A_{i n}+2 I_{\text {in }}+K_{A}\left(D_{i n}-I_{i n}\right)$, which can then be used to calculate the concentrations of all the other species involved in the activation reaction using Eq. 7. While the initial amounts of initiator $I_{i n}$ and activator $D_{\text {in }}$ are known from the reaction recipe, the initial amount of acids was set equal to that of acidic impurities estimated from HPLC-MS analysis of the cyOBT raw material. According to the patents, the cyOBT was originally synthesized by Cyclics Corp. involving the precipitation of residual acids, which indeed showed about $0.6 \%$ terephthalic acid 
by HPLC area. The value of the equilibrium constant $K_{a}$ can then be estimated by fitting the experimental values of $R / I_{\text {in }}$ at different activator contents, $D_{\text {in }} / I_{\text {in }}$. Increasing the latter ratio shifts the equilibrium of activation outlined in Eq. 8 towards the products, and thus increases the concentration of activated $\mathrm{Sn}(\mathrm{Oct})_{2}$. Consequently, conversion rate will increase until complete activation $\left(R=I_{\text {in }}\right)$ is established. This behavior is illustrated by plotting Eq. 6 for different levels of activation, where the increase of $D_{\text {in }} / I_{\text {in }}$ leads to steeper slopes $k_{P}{ }^{*} R$. Figure 3 shows that complete activation can be assumed at $D_{i n} / I_{\text {in }} \geq 200$, and the analytical solution has a best fit by using $K_{A}=0.028$. Using this value, the resulting level of activation for $D_{\text {in }} / I_{\text {in }}$ $=15$, as used in this work, was found to be of about $21 \%$.

With the known level of initiator activation, the actual concentration of active chains $R$ can be calculated. Then, Eq. 6 delivers $k_{P}{ }^{*}$, the rate constant of propagation, which we will discuss in the following.
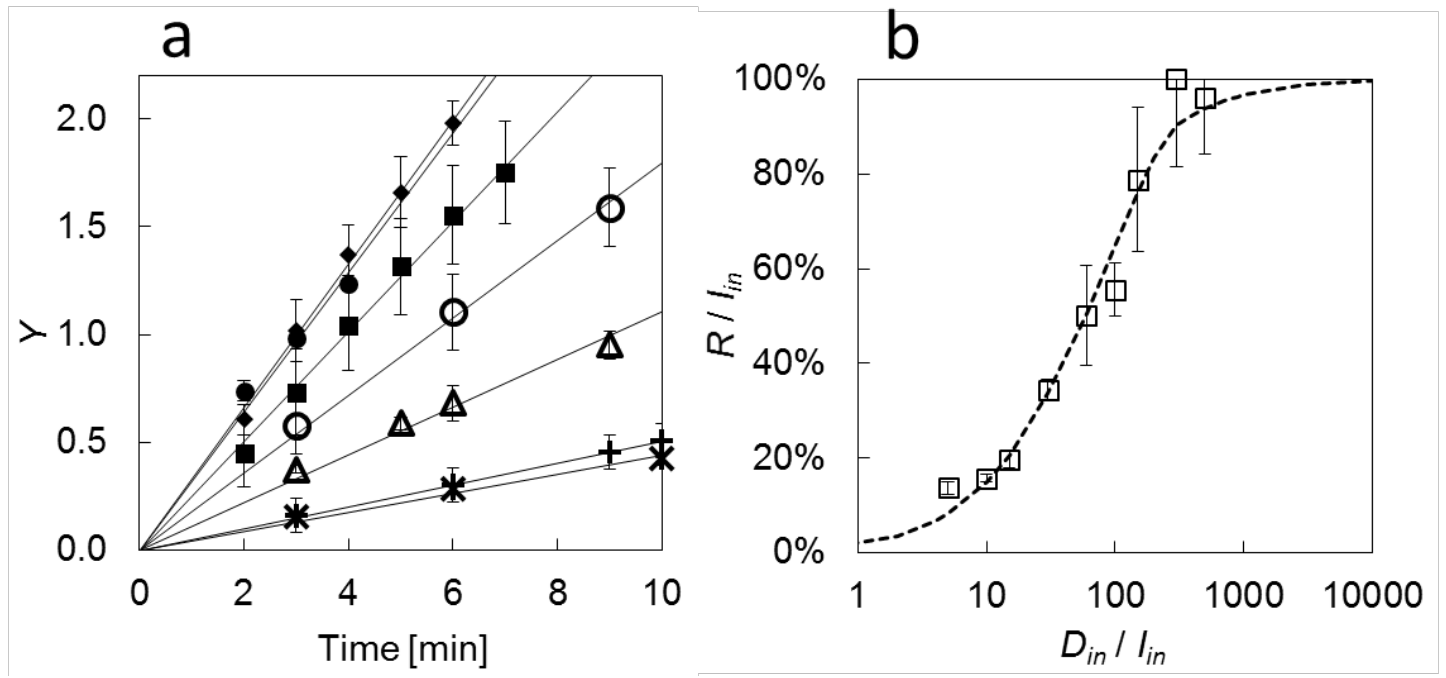

Figure 3. a, logarithmic conversion of ROP synthesis for $\mathrm{PBT}$ at $230^{\circ} \mathrm{C}$ using activator/initiator $D_{\text {in }} / I_{\text {in }}$ ratios of $5(+), 15(\times), 30(\Delta), 100(\circ), 150(\mathbf{\bullet}), 200(\bullet), 300(\bullet) . \mathbf{b}$, levels of initiator activation ( $\square$ ) for $0.1 \% \operatorname{Sn}(\mathrm{Oct})_{2}$ as function of $D_{\text {in }} / I_{\text {in }}$, based on the slopes derived from a, compared with the analytical solution for active initiator $R$ based on Eq. 9 with fitted $K_{A}$. Some data are not presented in the left graph to reduce visual entropy. 


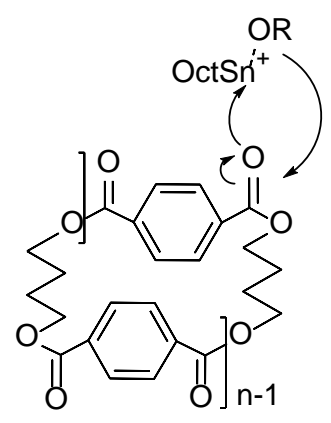

$C_{\mathrm{n}}$

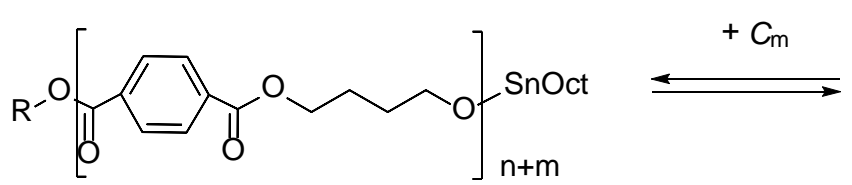

PBT
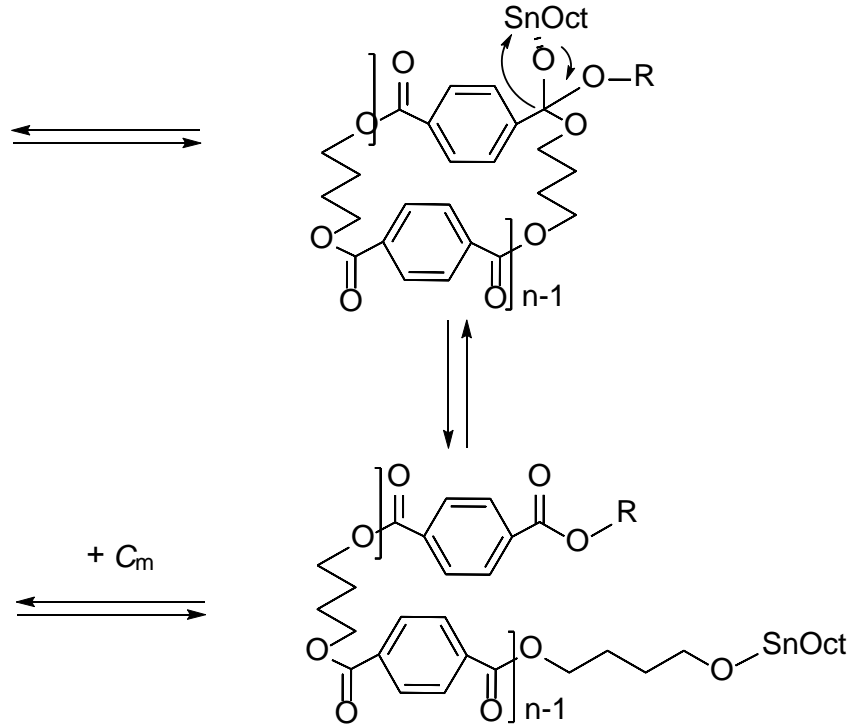

Figure 4. Ring-opening and propagation in Sn(Oct) $)_{2}$-based ROP for the synthesis of PBT.

\section{Ring-Opening, Propagation and Monomer Conversion}

The propagation using tin alkoxide typically follows a coordination-insertion mechanism: the exocyclic oxygen coordinates to the metal of the catalyst, after which carboxylic bond breakage leads to the opening of the ring, as shown in Figure 4. The active initiator site binds to the aliphatic group and the alcoholic ligand binds to the acid. Cyclic oligomers of size $n$ are then incorporated into the polymer chains via the same mechanism at the active Sn-site, adding $n$ repeat units to the resulting chain. The propagation reaction can be sketched as:

$$
R_{n}+C_{j} \underset{k_{P 2, j}}{\stackrel{k_{P, j}}{\rightleftarrows}} R_{n+j},
$$

where $C_{j}$ is a cyclic oligomer with $j$ repeat units and $R_{n}$ a growing chain with active Sn-site of length $n$ (in the case of $n=0, R_{0}$ indicates the active initiator itself). 
In all cases, the value of the corresponding rate coefficient was assumed to be independent of the polymer chain length. Thus, also the very first propagation step which involves the ringopening by means of the initiator $\left(R_{0}\right)$ was supposed to occur at the same rate. This assumption is based on the fact that reaction velocities were thought to mainly depend on the reactivity of end-groups which were the same for all reactive chains. Moreover, the propagation step was assumed to be irreversible for all cyclic oligomers: since the experimental results showed high monomer conversion, the reaction equilibrium was assumed to lie far on the product side (polymer), i.e. the forward reaction constant $\left(k_{P, j}\right)$ was much higher than its reverse $\left(k_{P 2, j}\right)$ for any $j$ and, therefore, the latter was neglected.

Besides the nature of the reactive polymer chain, the characteristics of the cyclic oligomers influence conversion. More specifically, the dependence of propagation rate on the cyclic oligomer size can be approached in two ways, using size-dependent and size-independent rate constants, both approaches having their supporting arguments, as outlined in the following. Firstly, we will consider the latter approach as pursued in the previous section to determine initiator activation, which lumps the reactivity of the different cyclic oligomers into one rate constant, independent of their size. This methodology can also be found in the literature, where propagation alone was considered as ROP mechanism for the synthesis of PBT[24], disregarding the molecular weight distribution. This situation can be envisioned where the mobility of the active site on the growing polymer chain is significantly reduced compared with that of the cyclic oligomer itself, which makes the different number of carbonyl groups in the cycle sterically equally available to the active site in the polymer chain. The corresponding material balance then reads as:

$$
\frac{d C_{j}}{d t}=-k_{P}^{*} C_{j} R \text {, }
$$


where $R$ is the total concentration of active chains and $k_{P}{ }^{*}$ represents the effective rate coefficient in the case of ring size-independent reactivity. This equation is therefore fully consistent with Eq. 5, which is quickly reobtained by multiplying both terms of Eq. 12 by $j$ and summing up for all $j$ values. For this simplified approach, $k_{P}{ }^{*}$ can easily be estimated from a regression on logarithmic conversion data using Eq. 6 and a regression of the slope in the linear region, as previously observed in Figure 2. From the regressions shown in Figure 5, a $k_{P}{ }^{*}$ value of $73.3 \mathrm{~L} \mathrm{~mol}^{-1} \mathrm{~min}^{-1}$ was obtained for the ring size-independent model.
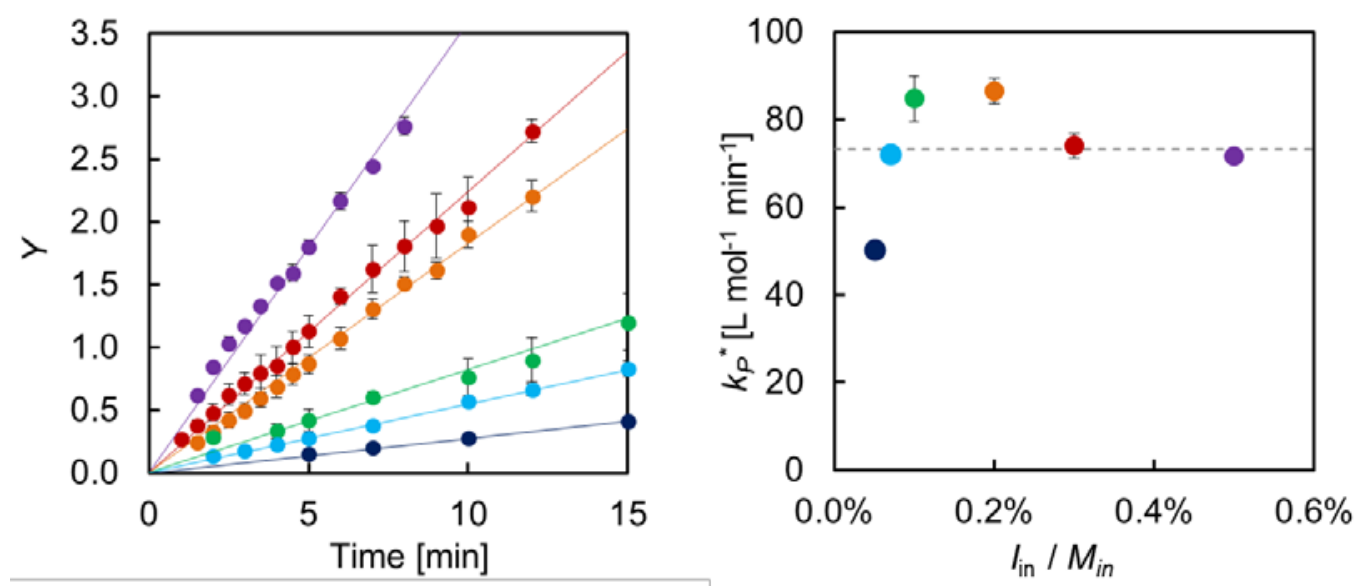

Figure 5. Estimation of $k_{P} *$ from ROPs at $230^{\circ} \mathrm{C}$ with a constant activator ratio $D_{\text {in }} / I_{\text {in }}=15$, varying the molar initiator concentrations $I_{i n} / M_{\text {in }}$ from $0.05 \%$ to $0.50 \%$. The obtained $k_{P}{ }^{*}$ values of each experiment are shown on the right, with the dashed line indicating the average value $k_{P}^{*}=73.3 \mathrm{~L} \mathrm{~mol}^{-1} \mathrm{~min}^{-1}$.

On the other hand, assuming that the availability of catalytic sites is not limiting, the reaction rate for propagation, which occurs through coordination of the catalytic metal site to carbonyl groups, is dependent on the number of such reactive groups in the cyclic oligomers, and therefore on their size. The population balance for cyclic oligomer $\left(C_{j}\right)$ consumption through size-dependent propagation then becomes: 


$$
\frac{d C_{j}}{d t}=-k_{P, j} C_{j} R=-k_{P} j C_{j} R
$$

where $k_{P}$ is now the rate coefficient representing coordination to carbonyl-bearing repeat units, with cycles $C_{j}$ incorporating $j$ of the latter. A fit using the population balance based on Eq. 12 to the data presented in Figure 5 yields $k_{P}=24.3 \mathrm{~L} \mathrm{~mol}^{-1} \mathrm{~min}^{-1}$. Not surprisingly, the estimated value for $k_{P}$ is about $1 / 3$ of $k_{P}{ }^{*}$, since the "weight-average ring size" given by the initial cyOBT distribution shown in Figure $\mathbf{1}$ is 3.28. This offset can be understood mathematically by calculating Eq. 12 back to monomeric repeat units as before. Applying the definition of $M$ as the sum of all repeat units incorporated in cycles in Eq.4, we obtain:

$$
\frac{d M}{d t}=-k_{P} \sum j^{2} C_{j} R=-k_{P} \bar{j} M R
$$

where

$$
\bar{j}=\frac{\sum j^{2} C_{j}}{\sum j C_{j}}
$$

which represents the weight-average ring size leading to the observed offset in rate constant between the two models, and is changing over time with the individual cycle's concentrations, starting at 3.28.

Then, let us use the two models, size-dependent and size-independent, to predict the evolutions of conversion, residual concentration of each cyclic oligomer and of the overall amount of repeating units, and the number average molecular weight, $M_{n}$, using the values of propagation rate constants estimated above. The predictions of the two models are compared in Figure 6. The agreement of the both models with the experimental data of conversion and numberaverage molecular weight for two different data sets using one single rate constant value is appreciable, as shown in Figure 6a and $\mathbf{d}$. At larger conversions, the size-independent model 
predicts strictly linear behavior of the logarithmic conversion defined by Eq. 6, where the sizedependent model exhibits minor deviation. This behavior is explained in Figure 6c, which shows the different predictions in terms of cyclics or monomer concentration profiles, compared with the cyclic oligomer consumption from higher resolution SEC previously presented in Figure 5b. The deviation from linearity by the size-dependent model reflects the changing reactivity of the cyclic mixture along the reaction (larger rings are consumed faster than smaller ones), where the size-independent model assumes constant reactivity of all species. The time evolution of the overall concentration of repeating units $(M)$ predicted by the sizeindependent model is practically superimposed with that of the ternary cycle, $C_{3}$, due to the weight-average ring size $\bar{\jmath}$ of around 3. Judging from the experimental data in Figure 6c, the actual reactivity of different ring sizes seems to be very similar, suggesting that $\bar{J}$ is almost constant over the course of the reaction. While more experiments would be required to make a robust and conclusive discrimination between the two models, for this system the prediction power of both models is practically the same. Therefore, the simpler size-independent approach for propagation will be used in the following discussion. 
a
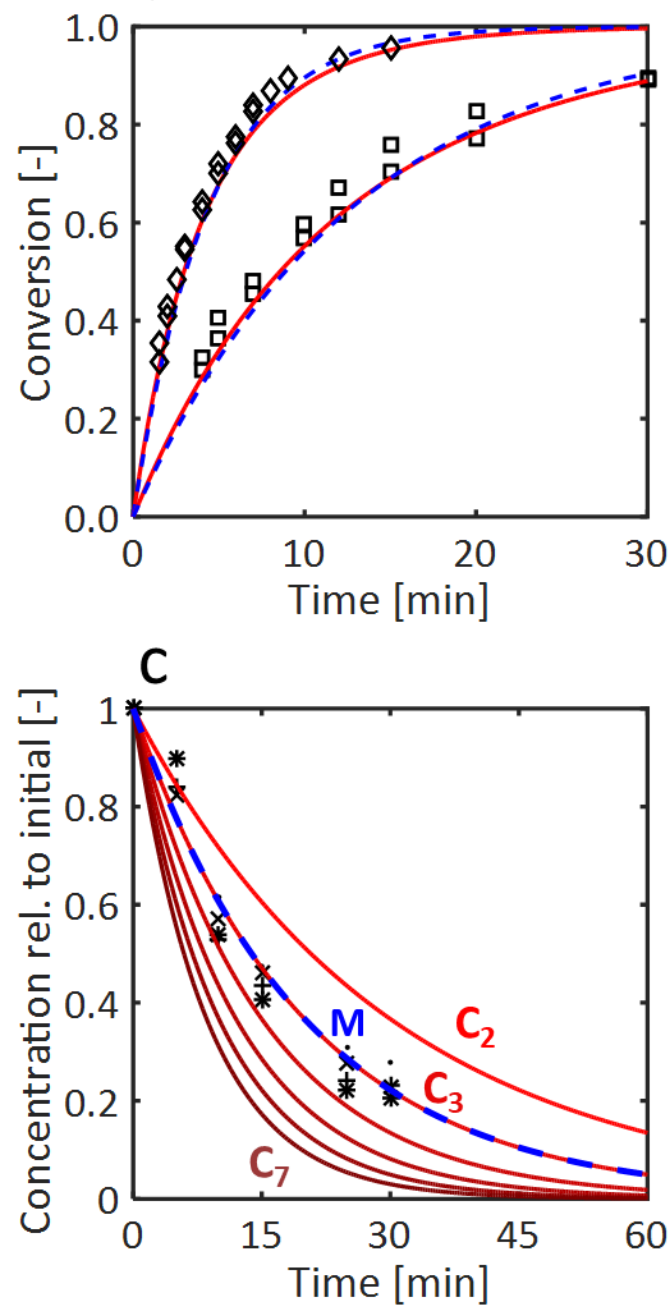

b
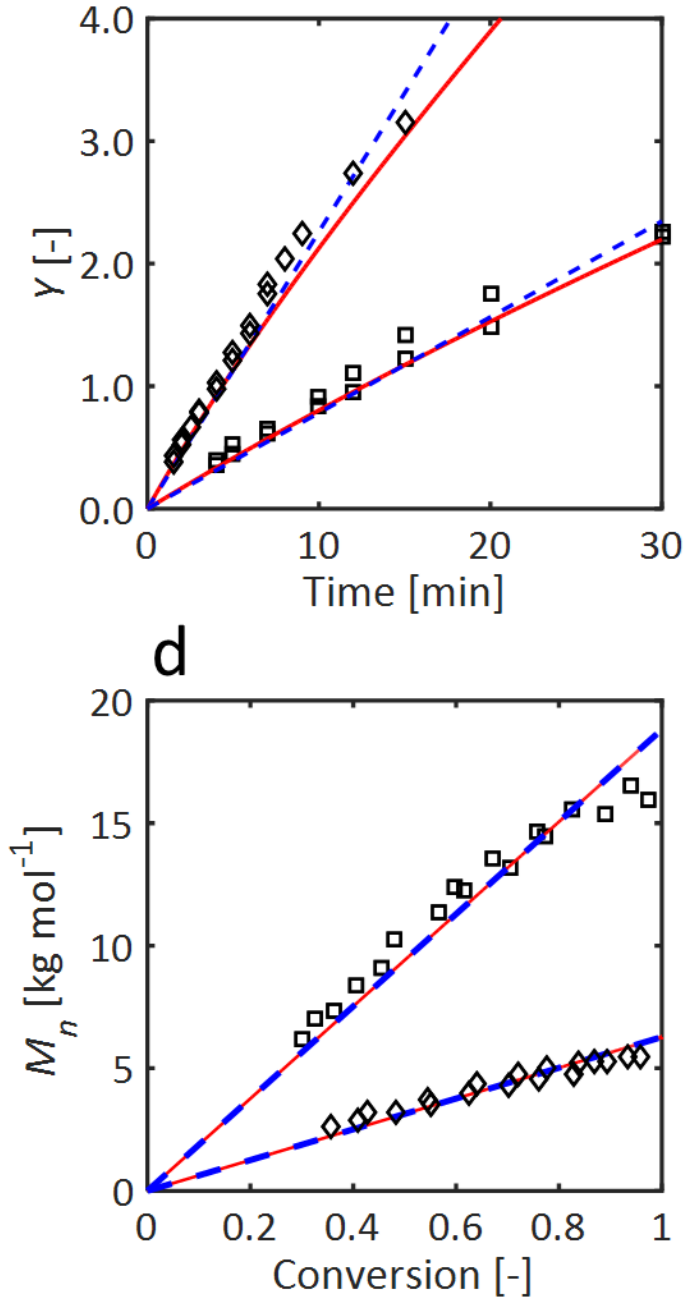

Figure 6. Conversion (a), logarithmic conversion (Eq. 6) (b) and number-average molecular weight (d) of ROP for PBT using $0.3 \%(\diamond)$ and $0.1 \%$ ( $\square)$ Sn(Oct $)_{2}$, compared with the predictions of two different kinetic models based on ring size-dependent reactivity (-) and sizeindependent reactivity (---). (c) shows the time evolution of the predicted concentrations of cyclic oligomer species $C_{X}$ from the size-dependent model, and the lumped monomer $M$ concentration derived from the size-independent model, compared with experimental conversion data previously shown in Figure $5 \mathbf{b}$ for the individual ring sizes $C_{2}(\times), C_{3}(\cdot), C_{4}$ (*), $C_{5}(+)$. In all cases, $D_{\text {in }} / I_{\text {in }}$ of $15: 1$ was applied. 


\section{Chain Transfer, Transesterification and Molecular Weight Evolution}

While the reaction rate may be affected by how the reactivity of the cyclic monomers is taken into account, the molecular weight evolution is not affected by either propagation model used in this study. As shown in Figure 6d, molecular weight progression relative to conversion is independent on the rate law selected for the propagation step because the incorporation of the corresponding number of repeat units per oligomer into polymer chains is conserved. On the other hand, the molecular weight distribution is mainly determined by the other typical ROP reaction mechanisms, such as chain transfer and transesterification.

Reversible chain transfer describes the reaction between the active site of a growing chain with any free terminal hydroxyl group, which leaves the previously active chain dormant while the recipient of the initiator becomes active. This mechanism is sketched in Figure 7 and the reaction can be written as

$$
R_{n}+D_{i} \underset{k_{C T}}{\stackrel{k_{C T}}{\rightleftarrows}} R_{i}+D_{n}
$$

where $R_{n}$ is an actively growing polymer chain of length $n$, and $D_{i}$ is a dormant chain of length $i$. The special case of $D_{0}$ represents an OH-bearing species with zero repeat units, which in our case is the activator 1-dodecanol. Chain transfer does not affect the system reactivity (the number of active growing chains remains the same) but greatly affects the average molecular weight. Through chain transfer, any $\mathrm{OH}$-bearing species in the system, such as $D_{0}$ or other polymer chains, can be activated by the initiator and grow through propagation, which decreases the overall chain length by enlarging the denominator in the following equation:

$$
M_{n}=\frac{M_{i n} X}{P} m_{r u}=\frac{M_{i n} X}{R+D} m_{r u} \text {, }
$$


where $P$ is the total concentration of chains in the system, which is the summation of active chains $R$ and dormant chains $D$. The rate constant of this reaction step was assumed independent upon the direction, i.e. the corresponding equilibrium constant is equal to 1 .

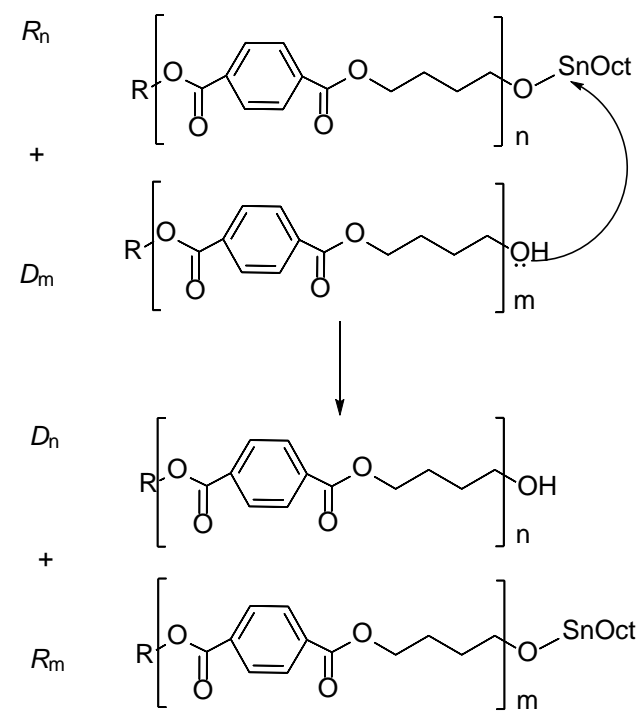

Figure 7. Chain transfer of initiator from active to dormant chain during Sn(Oct) $)_{2}$-based ROP

With sufficiently fast chain transfer compared with propagation, the dispersity of the molecular weight distribution $Ð=M_{w} / M_{n}$ would approach 1 . Since in ROP a broader dispersity around 2 is usually observed, another mechanism has to be taken into account: transesterification describes the reaction of an active polymer chain with any carbonyl group within another chain, which may result in two species of lengths potentially very different than before. This reshuffling of the chain lengths does not affect $M_{n}$ but greatly influences the weight-average molecular weight $M_{w}$ and thus $Ð$. The reaction can be sketched using the previously introduced active chains $R$ and dormant chains $D$ as:

$$
R_{i}+R_{j} \underset{k_{T r}}{\stackrel{k_{T r}}{\rightleftarrows}} R_{i+j-n}+R_{n}
$$




$$
R_{i}+D_{j} \underset{k_{T r}}{\stackrel{k_{T r}}{\rightleftarrows}} R_{i+j-n}+D_{n} .
$$

This reaction scheme assumes "regular" transesterification, conserving the ratio of diacid and glycol in the repeat units. However, during transesterification of a symmetric diacid such as the terephthalic acid used herein, which has two attackable carbonyl sites, also so-called "disproportionation" of the diacid and diol subunits may occur. This would lead to "doubleactive” and deactivated chains. Although the chains born from these two transesterification pathways are different, and so are their further reaction kinetics, computational studies indicated a negligible dissimilarity between these two mechanisms in their influence on the $M_{n}$ and $M_{w}$ progression[15]. As a consequence, for kinetic modelling in the first instance, disproportionation was disregarded in this work. Both mechanisms are compared in Figure 8. 
a
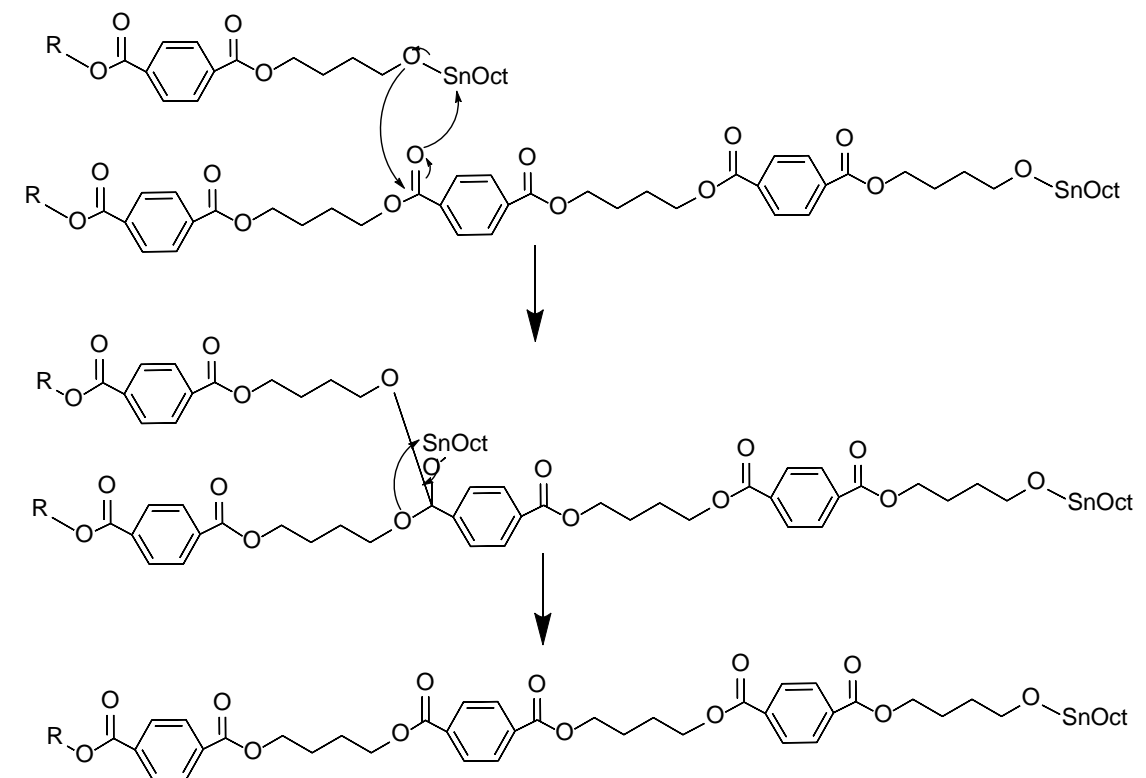

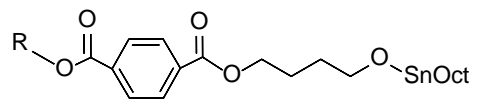

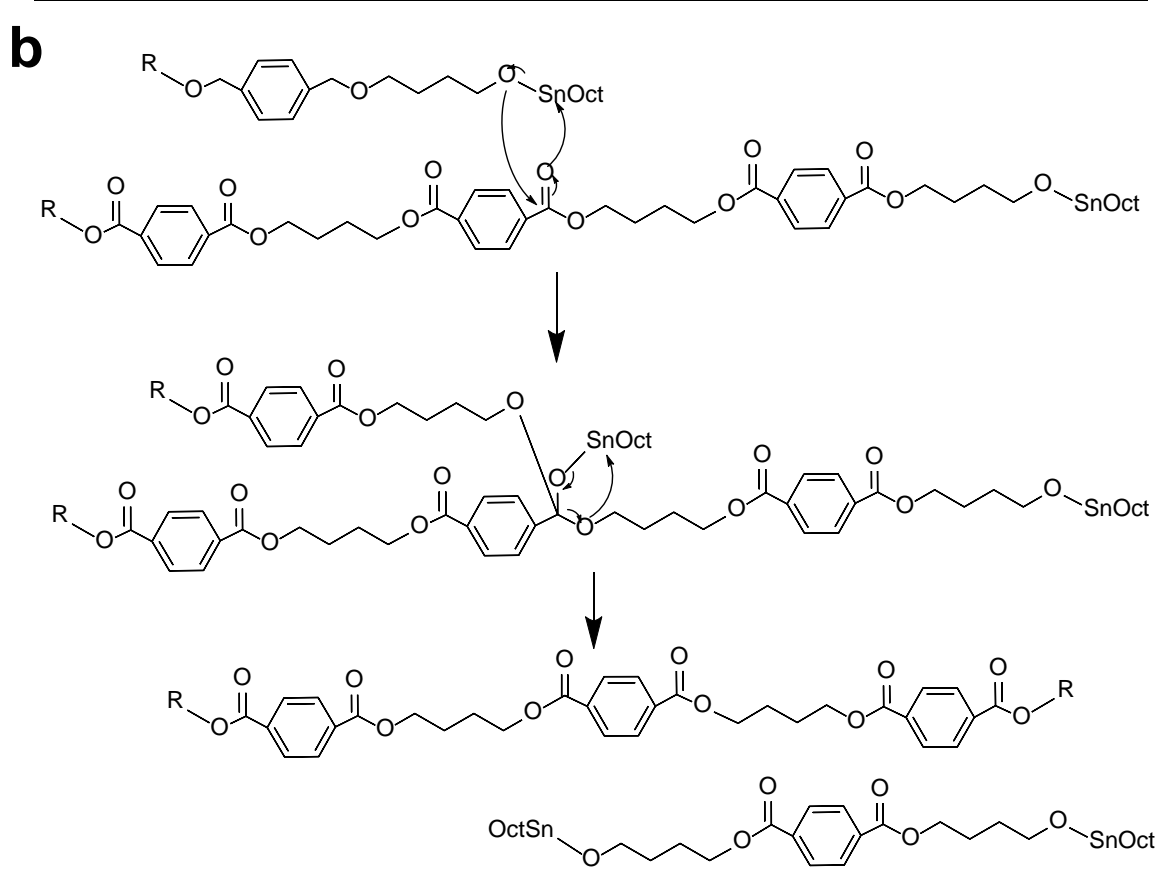

Figure 8. Transesterification reactions for PBT involving two active chains forming (a) two active chains of different length through "regular" transesterification, and (a) one doubleactive and one dormant chain through disproportionation. 
Considering all the reactions discussed above, the population balances for active and dormant chains can be written as follows:

$$
\begin{aligned}
& \frac{d R_{n}}{d t}=k_{P} M R_{n-1}-k_{P} M R_{n} \\
& +k_{C T} D_{n} \sum_{i=0}^{\infty} R_{i}-k_{C T} R_{n} \sum_{i=0}^{\infty} D_{i} \\
& +k_{T r}\left(\begin{array}{c}
\sum_{i=0}^{\infty} R_{i} \sum_{j=n+1}^{\infty} R_{j}+\sum_{i=0}^{n-1} R_{i} \sum_{j=n-i}^{\infty} R_{j}-R_{n} \sum_{j=0}^{\infty} j R_{j} \\
-n R_{n} \sum_{i=0}^{\infty} R_{i}+\sum_{i=0}^{\infty} R_{i} \sum_{j=n+1}^{\infty} D_{j}-R_{n} \sum_{j=0}^{\infty} j D_{j}
\end{array}\right) \\
& \frac{d D_{n}}{d t}=-k_{C T} D_{n} \sum_{i=0}^{\infty} R_{i}+k_{C T} R_{n} \sum_{i=0}^{\infty} D_{i} \\
& +k_{T r}\left(-n D_{n} \sum_{i=0}^{\infty} R_{i}+\sum_{i=0}^{n-1} R_{i} \sum_{j=n-i}^{\infty} D_{j}\right)
\end{aligned}
$$

The population balances for the active chains (Eq. 19) incorporate terms for chain growth via propagation, activation and deactivation through chain transfer to dormant chains, and the various transesterification combinations of active chains with active and dormant chains. Dormant chains (Eq. 20) cannot undergo propagation, but participate in the polymerization through chain transfer and transesterification. Note that when $n=0$, these equations also include the population balances for the activated initiator $R_{0}$ and the alcohol $D_{0}$, respectively. This results in a non-defined term (precisely, the first term) for active chain formation through propagation, which can be treated with the Kroenecker-delta; however, since the model will be solved using the moments only, this term is treated during moment derivation[14]. Also note that Eq. 19 for $n<8$ is only defined for cyclic species with size $j<7$; however, it can be shown that the combination of the possible cases results in the same final moment equations, as well. 
To solve the overall population balance, the method of moments was applied[14], which offers much faster calculations compared with complete population balance solving due to the much more limited number of equations. This approach is expected to be fully adequate because of the monomodal nature of the distributions experimentally measured. Given the moments of the different species, active and dormant chains, number average molecular weight $\left(M_{n}\right)$ and weight average molecular weight $\left(M_{w}\right)$ can be readily calculated:

$$
\begin{gathered}
M_{n}=\frac{\sum_{n=0}^{\infty} n\left(R_{n}+D_{n}\right)}{\sum_{n=0}^{\infty}\left(R_{n}+D_{n}\right)}=\frac{\lambda_{1}+\mu_{1}}{\lambda_{0}+\mu_{0}} \\
M_{w}=\frac{\sum_{n=0}^{\infty} n^{2}\left(R_{n}+D_{n}\right)}{\sum_{n=0}^{\infty} n\left(R_{n}+D_{n}\right)}=\frac{\lambda_{2}+\mu_{2}}{\lambda_{1}+\mu_{1}}
\end{gathered}
$$

where $\lambda_{j}$ and $\mu_{j}$ denote the moments of $j$-th order for active and dormant chains, defined as:

$$
\begin{aligned}
& \lambda_{j}=\sum_{n=0}^{\infty} n^{j} R_{n} \\
& \mu_{j}=\sum_{n=0}^{\infty} n^{j} D_{n}
\end{aligned}
$$

The resulting moment equations are summarized in Appendix. Combining Eq. 11 with such equations, the final system of model equations is obtained, whose solution was performed numerically after setting the corresponding initial conditions. As explained earlier, in a living system without termination the number of active and dormant chains is conserved, therefore the $0^{\text {th }}$ moments for both chain species are constants over the reaction time. More precisely, $\lambda_{0}$ 
$=R_{0}$ and $\mu_{0}=D_{\text {in }}-R_{0}$; since at $t=0$ no polymer has formed yet, the remaining initial conditions were: $\lambda_{1}=\lambda_{2}=\mu_{1}=\mu_{2}=0$.

The molecular weight evolutions are then readily obtained using these moment equations with the appropriate rate constants for chain transfer and transesterification, whose evaluation will be discussed in the following. Focusing first on the number average molecular weight $M_{n}$, we can derive several insights into the governing reaction mechanisms. As shown in Figure 9 and earlier in Figure 6d, livingness is again observed as a linear behavior of $M_{n}$ vs. conversion, which demonstrates that growing chains (active and dormant) are formed immediately after the reaction start, and their number does not change later on during the living stage. Moreover, the observed moderate molecular weights prove that chain transfer to free $\mathrm{OH}$-groups is significant, since otherwise extremely high molecular weights would be expected, as obvious from Eq. 16, comparing the cases $D=0$ and $D=D_{i n}$, respectively.
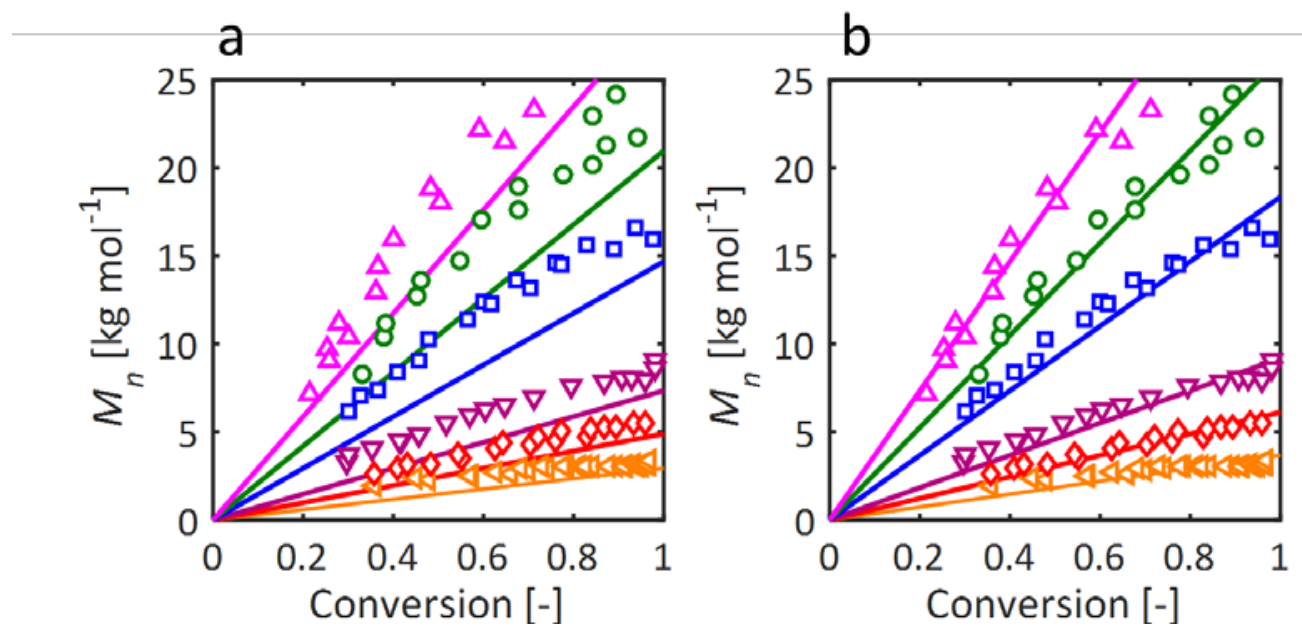

Figure 9. Experimental number average molecular weight $M_{n}$ of PBT-ROP at $230^{\circ} \mathrm{C}$ using $0.05 \%(\Delta), 0.07 \%(\circ), 0.1 \%(\square), 0.2 \%(\nabla), 0.3 \%(\diamond), 0.5 \%(\triangleleft)$ SnOct $_{2} / 15: 1 D_{\text {in }}$, compared with model predictions applying $0 \%$ (a) and 20\% (b) loss of 1-dodecanol $D_{\text {in }}$. 
On the other hand, a constant underprediction of $M_{n}$ using the exact values of the experimental recipe in the model can be observed in Figure 9a. This observation indicates that actually fewer chains than assumed are present in the system: since $M_{n}$ is most dominantly influenced by the number of dormant chains $D$ in the case of the $\operatorname{Sn}(\mathrm{Oct})_{2}$-initiated system, due to the large excess of activator (1-dodecanol, $D_{\text {in }}$ ), a portion of these OH-bearing dormant chains must therefore be unavailable for chain transfer. Looking at Eq. 7, the sum in the denominator of Eq. 16 is no other than $D_{i n}$, whose value can be reduced as a result of two separate observable effects. The residual acids present in the cyOBT raw material after their synthesis, as discussed earlier, may well neutralize a portion of the alcoholic species, thus leading to higher molecular weights than expected. Another aspect is the partial evaporation of dodecanol: upon injection of the initiator solution, a small vapor cloud was usually formed, that may have been 1-dodecanol due to the significant vapor pressure at the given reaction temperature $\left(230^{\circ} \mathrm{C}, 1\right.$-dodecanol boiling point $259^{\circ} \mathrm{C}$ ). NMR analysis of samples taken at and shortly after the injection of initiator into the hot molten cyOBT at $230^{\circ} \mathrm{C}$ confirmed the immediate loss of 1-dodecanol at up $20 \%$. The amount of available 1-dodecanol for chain transfer was reduced accordingly in the model ( $D_{\text {in }} / I_{\text {in }}=12$ instead of 15 as prepared experimentally), resulting in an appreciable fit of the predicted $M_{n}$ with the other experimental data, as shown in Figure $\mathbf{9 b}$.

Having understood the conditions to predict number-average molecular weights, the description of weight-average molecular weights $M_{w}$ and polydispersity $Ð$ is the most challenging task. For $M_{n}$, the linear trend indicated that chain transfer simply has to be relatively fast compared with propagation. In fact, a slow chain transfer would have resulted in an increasing number of growing chains along the course of reaction, observable as a bend towards lower molecular weights. $M_{w}$ and $\oslash$, however, are both affected by an interplay of chain transfer and transesterification. In order to elucidate such interplay, a model sensitivity analysis was carried out whose results are shown in Figure 11 with reference to a specific reaction $(0.3 \%$ $\left.\mathrm{Sn}(\mathrm{Oct})_{2} / 1: 15 D_{\text {in }}\right)$. As shown in Figure 10a and 11c, the experimental data exhibit dispersities 
reaching values of 1.6-1.8 already after 30\% conversion, progressing to the expected value of 2 for ROP towards complete conversions. These results indicate that transesterification has to be sufficiently fast compared with chain transfer, where the latter cannot be assumed simply very large as for other polymer systems[22]. Figure 10a and 11c also show how the corresponding rate constants $k_{C T}$ and $k_{T r}$ have superimposed effects on the dispersity: faster chain transfer counterbalances the increasing dispersion of molecular weight by transesterification. Three regimes of reaction can be identified: an initially sharp increase of dispersity due to few rapidly growing and reshuffling chains, after which chain transfer becomes dominant to homogenize the molecular weights until eventually the polymer chains are long enough to offer as many attackable positions for transesterification to become dominant. A fit to the data of ROP using $0.3 \% \operatorname{Sn}(\mathrm{Oct})_{2} / 1: 15 D_{\text {in }}$ resulted in the optimized values $k_{C T}=1000 \mathrm{~L} \mathrm{~mol}^{-1} \mathrm{~min}^{-1}$ and $k_{T r}=24 \mathrm{~L} \mathrm{~mol}^{-1} \mathrm{~min}^{-1}$ as shown in Table 2 . These values complete the set of parameters to be determined for this kinetic model, which could then successfully be validated against other experiments at different initiator concentrations at $230^{\circ} \mathrm{C}$, as shown in Figure 11. 

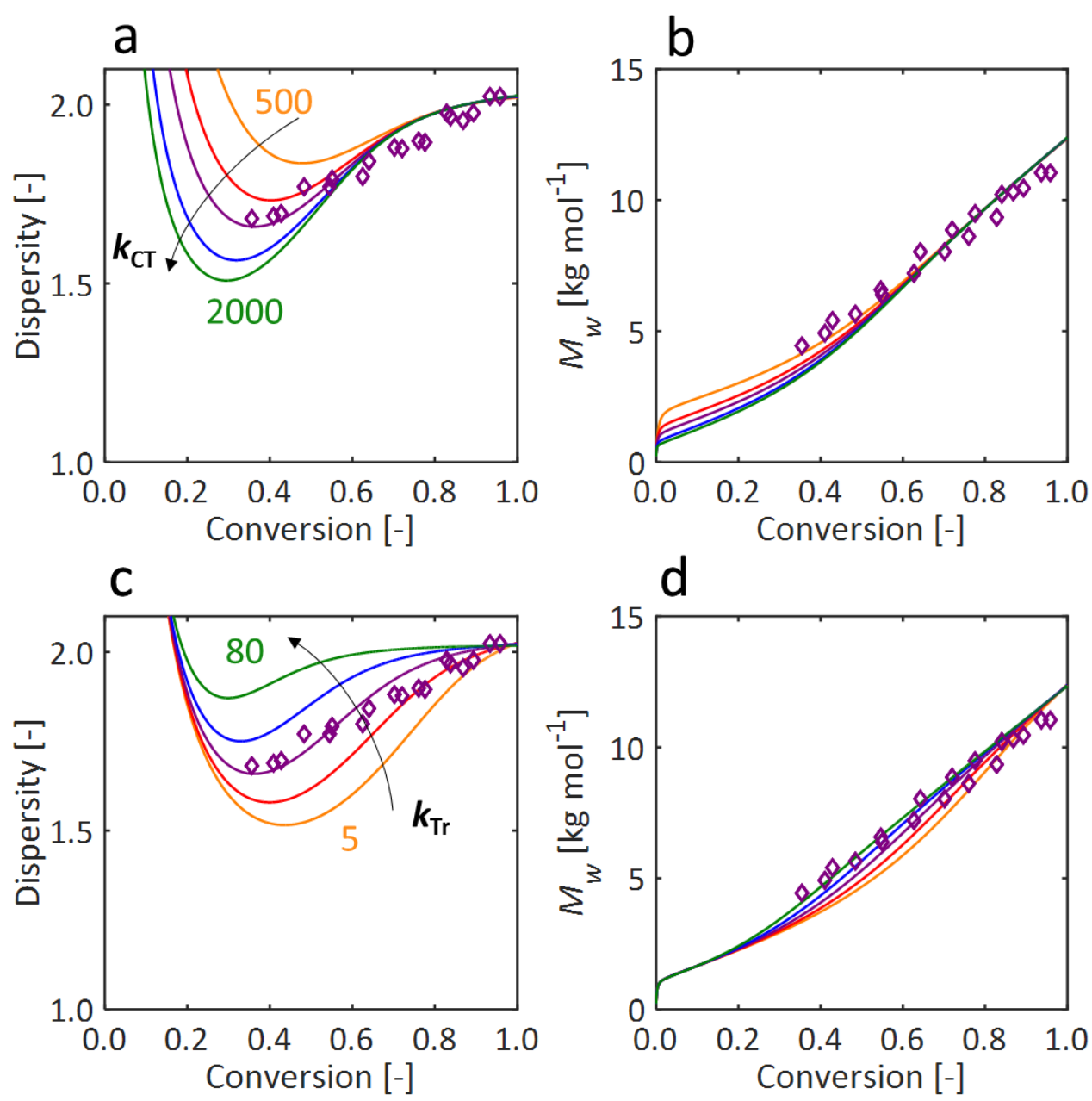

Figure 10. Sensitivity analysis of the kinetic model to variation of the rate constants for chain transfer $\left(k_{C T}\right)$ and transesterification $\left(k_{T r}\right)$. Their effect on the model predictions are compared with the experimental data of ROP using $0.3 \%$ SnOct $_{2} / 1: 15 D_{\text {in. }}$. The graphs show the effect of $k_{C T}=500,750,1000,1500,2000 \mathrm{~L} \mathrm{~mol}^{-1} \mathrm{~min}^{-1}$ on dispersity (a) and weight-average molecular weight (b), as well as the same effects based on the transesterification coefficient $k_{T r}=10,15$, 24, 40, $80 \mathrm{~L} \mathrm{~mol}^{-1} \mathrm{~min}^{-1}$ (c, d). During these variations, the other bespoken parameters were held constant at the values indicated by the purple lines in the plots, which show the values used in the final version of the optimized model. 

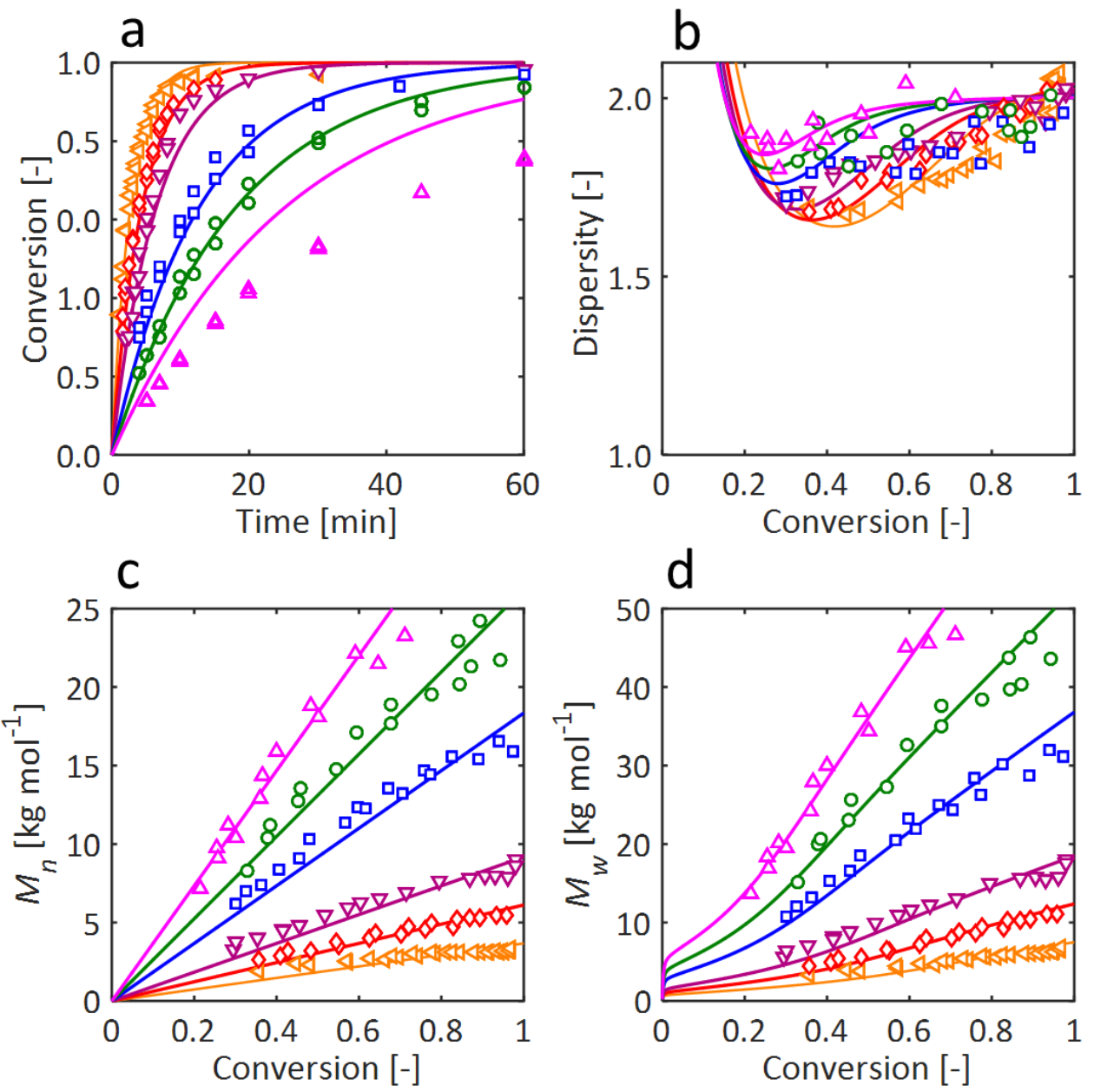

Figure 11. Comparison of conversion, dispersity, and average molecular weights $\left(M_{n}\right.$ and $\left.M_{w}\right)$ results from experiments and the optimized kinetic model of ROP for the synthesis of PBT at $230^{\circ} \mathrm{C}$ using $0.05 \%(\Delta), 0.07 \%(\odot), 0.1 \%(\square), 0.2 \%(\nabla), 0.3 \%(\diamond), 0.5 \%(\triangleleft)$ SnOct $_{2} / 1: 15 D_{\text {in. }}$ The two ROPs at the lowest initiator concentrations were performed using 25\% (mass per mass cyOBT) tetraglyme to enhance plasticization and reduce diffusion limitations, which were apparent in the previous non-plasticized experiments. 
Table 2. Parameters and conditions used in the ring size-independent kinetic model for Sn(Oct) $)_{2}$-based ROP for PBT at $230^{\circ} \mathrm{C}$.

\begin{tabular}{llll} 
& Value & Use & Determination \\
\hline$k_{P}{ }^{*}$ & $73.3 \mathrm{~L} \mathrm{~mol}^{-1} \mathrm{~min}^{-1}$ & Propagation constant & Fit to $Y$ \\
$k_{C T}$ & $1000 \mathrm{~L} \mathrm{~mol}^{-1} \mathrm{~min}^{-1}$ & Chain transfer rate constant & Fit to $Ð, M_{w}$ \\
$k_{T r}$ & $24 \quad \mathrm{~L} \mathrm{~mol}^{-1} \mathrm{~min}^{-1}$ & Transesterification rate constant & Fit to $\oslash, M_{w}$ \\
$D_{i n, \text { loss }}$ & $20 \%$ & Molecular weight calculation & NMR, SEC
\end{tabular}

\section{Temperature dependence}

In order to explore the temperature as another parameter inside the operating region of the reaction, a preliminary study on the influence and predictability of temperature was undertaken to estimate Arrhenius constants of propagation. Bearing in mind, that the level of activation and its equilibrium constant $K_{a}$ discussed in Section 3.2.1 may well be a function of temperature, the activation energy of propagation $E_{A, P}$ determined here is an activation-lumped approximation of the actual $E_{A, P}$ of propagation. To determine the dependence of the propagation rate constant $k_{P}{ }^{*}$ to temperature, a data set using $0.2 \% \mathrm{Sn}(\mathrm{Oct})_{2}$ at different temperatures was established and used for model development. Figure 12 pesents these data, where reaction rate increases with temperature as expected. The different $k_{P}{ }^{*}$ were determined as before by fitting the logarithmic conversion, which can then be plotted in logarithmic Arrhenius form (b) to yield a lumped activation energy $E_{\mathrm{A}}=59.0 \mathrm{~kJ} \mathrm{~mol}^{-1}$ and pre-exponential factor $A=1.14 \times 10^{8} \mathrm{~L} \mathrm{~mol}^{-1} \mathrm{~min}^{-1}$. As a first guess, chain transfer and transesterification constants $k_{C T}$ and $k_{T r}$ were normalized by the change in $k_{P}{ }^{*}$, assuming similar dependence on temperature, which causes the predictions for polydispersity to collapse in one single curve. The prediction quality in terms of conversion (c), dispersity (d) and average molecular weights (e, f) was appreciable, indicating the validity of this simplified approach. 
The activation energy for the $\mathrm{Sn}(\mathrm{Oct})_{2}$ initiator system used in this study is lower than that of butylchlorotin dihydroxide $\left(65.8 \mathrm{~kJ} \mathrm{~mol}^{-1}\right)$, which has been used for ROP in the literature, and significantly lower than the activation energy reported for conventional polycondensation using tetrabutoxytitanate, indicating that PBT synthesis via ROP can be much faster[24]. In industrial practice this is beneficial, since the polymerization of PBT is ideally run below its melting point $\left(190^{\circ} \mathrm{C}\right)$ to exploit simultaneous crystallization, which then requires the actual polymerization to be fast enough[24].
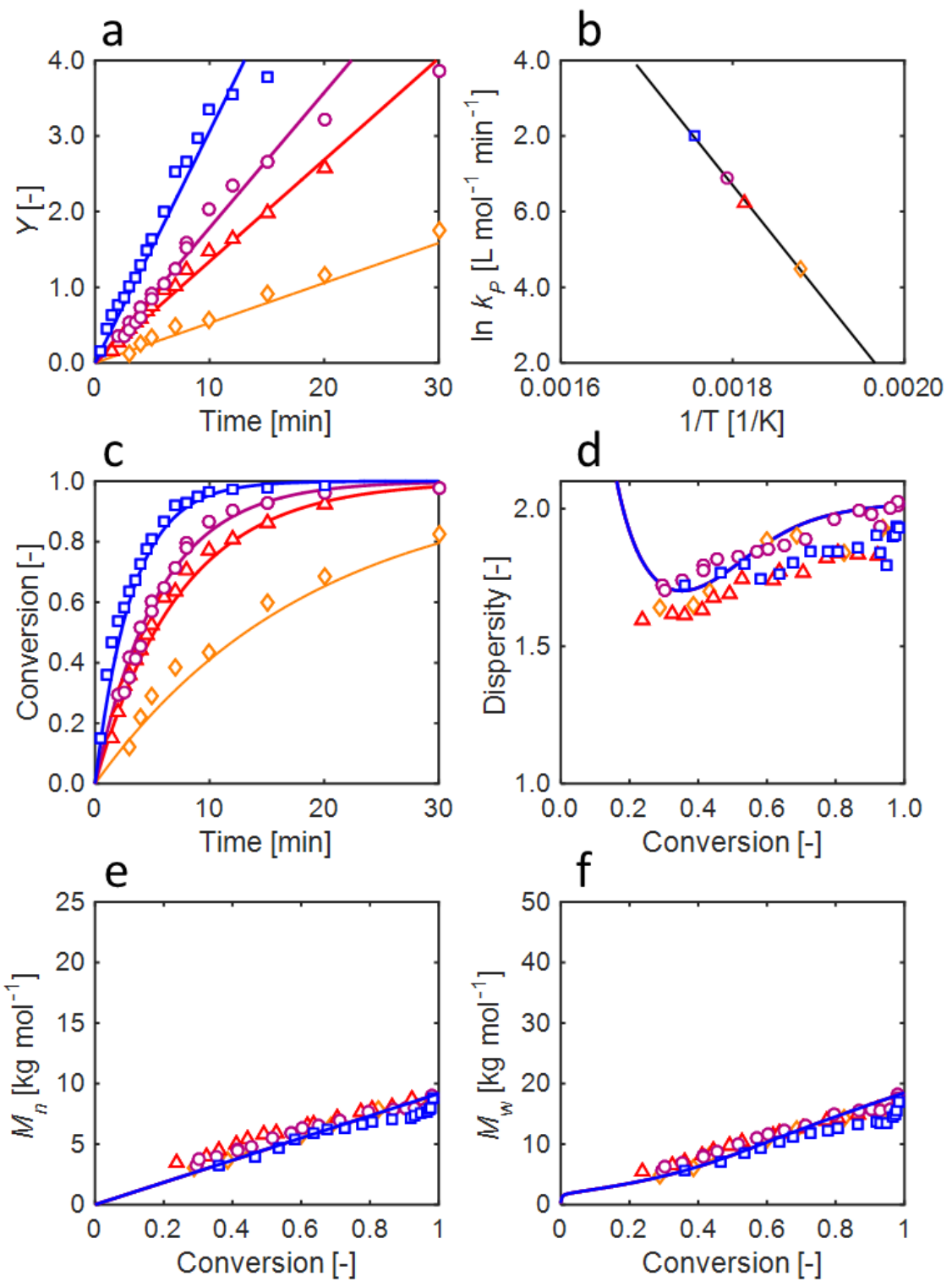
Figure 12. Logarithmic conversions of PBT ROP using $0.2 \% \operatorname{Sn}(\mathrm{Oct})_{2} / 1: 15 D_{\text {in }}$ to fit $k_{P}{ }^{*}$ at different temperatures (a): $250^{\circ} \mathrm{C}(\square), 230^{\circ} \mathrm{C}(\circ), 220^{\circ} \mathrm{C}(\Delta), 190^{\circ} \mathrm{C}(\diamond)$. The resulting Arrhenius parameters obtained from (b) were then used to predict the same kinetic data in terms of conversion (c), dispersity (d) and molecular weights (e, f) using the model based on a temperature-dependent $k_{P}{ }^{*}$.

\section{CONCLUSIONS}

Ring-opening polymerization (ROP), as an alternative polyester synthesis route to polycondensation, offers precise prediction of monomer conversion and molecular weights evolutions. The herein established kinetic model helps understanding and predict ROP of cyclic polyester oligomers on the example of polybutylene terephthalate synthesis using tin octoate as initiator at $230^{\circ} \mathrm{C}$. Since the cyclic oligomers appear as a distribution of ring sizes, the number of reactive carbonyl sites grows with the number of repeat units per ring, which can be described by a size-dependent propagation model. However, also simpler and size-independent conversion calculations deliver an appreciable fit to the experimental data, suggesting that not the accessibility of carbonyl sites but rather the active metal site on the polymer chain are ratedetermining. The molecular weight distribution is mainly affected by chain transfer and transesterification reactions, where the former enable $\mathrm{OH}$-bearing species in the system to act as chain starters. This affects directly the number average molecular weight, which follows a linear trend with conversion, confirming the living-like behaviour of ROP. Transesterification is the reaction responsible for a reshuffling of chain lengths, eventually leading to the typically observed dispersities of molecular weights around 2. Accounting for these reactions in a population balance model, the experimental data in terms of conversion and molecular weights 
can be fitted well with the three associated rate parameters where, notably, chain transfer cannot be assumed simply much faster than the other reactions. Besides the understanding of ROP for PBT polyester synthesis, the model developed herein is capable to extrapolate across the initiator concentration and reaction temperature, and provides a basis for the physically founded computational modelling of other common polyesters such as PET, PEF and PBF, as well.

\section{ACKNOWLEDGEMENTS}

This work was funded through project 16120.1-PFEN-IW by the Swiss Commission for Technology and Innovation (CTI). The valuable experimental work by Diana Hohl and Marcel Kröger is also gratefully acknowledged.

\section{REFERENCES}

[1] G. Kannan, S.E. Grieshaber, W. Zhao, Thermoplastic Polyesters, in: O. Olabisi, K. Adewale (Eds.), Handb. Thermoplast. Second Ed., 2015: pp. 319-346.

[2] H.R. Kricheldorf, M. a I. Masri, N. Lomadze, G. Schwarz, Telechelic poly(butylene terephthalate)s by means of bismuth catalysts, Macromolecules. 38 (2005) 9085-9090.

[3] F. Neaţu, G. Culică, M. Florea, V.I. Parvulescu, F. Cavani, Synthesis of Terephthalic Acid by p-Cymene Oxidation using Oxygen: Toward a More Sustainable Production of Bio-Polyethylene Terephthalate, ChemSusChem. $9 \quad$ (2016) 3102-3112. doi:10.1002/cssc.201600718.

[4] Y. Zhu, C. Romain, C.K. Williams, Sustainable polymers from renewable resources, Nature. 540 (2016) 354. doi:10.1038/nature21001. 
[5] A. Gandini, T.M. Lacerda, A.J.F. Carvalho, E. Trovatti, Progress of Polymers from Renewable Resources: Furans, Vegetable Oils, and Polysaccharides, Chem. Rev. 116 (2016) 1637-1669. doi:10.1021/acs.chemrev.5b00264.

[6] D. Pfister, G. Storti, F. Tancini, L.I. Costa, M. Morbidelli, Synthesis and Ring-Opening Polymerization of Cyclic Butylene 2,5-Furandicarboxylate, Macromol. Chem. Phys. 216 (2015) 2141-2146.

[7] E. De Jong, M.A. Dam, L. Sipos, G.J.M. Gruter, Furandicarboxylic acid (FDCA), A versatile building block for a very interesting class of polyesters, in: P. Smith (Ed.), ACS Symp. Ser., 2012: pp. 1-13. doi:10.1021/bk-2012-1105.ch001.

[8] F. Neaţu, G. Culică, M. Florea, V.I. Parvulescu, F. Cavani, Synthesis of Terephthalic Acid by p-Cymene Oxidation using Oxygen: Toward a More Sustainable Production of Bio-Polyethylene Terephthalate, ChemSusChem. 9 (2016) 3102-3112.

[9] S. Venkatachalam, S.G. Nayak, J. V Labde, P.R. Gharal, K. Rao, A.K. Kelkar, Degradation and Recyclability of Poly (Ethylene Terephthalate), in: H.E.-D.M. Saleh (Ed.), POLYESTER, InTech, 2012.

[10] A. Pellis, K. Haernvall, C.M. Pichler, G. Ghazaryan, R. Breinbauer, G.M. Guebitz, Enzymatic hydrolysis of poly(ethylene furanoate), J. Biotechnol. 235 (2016) 47-53. doi:10.1016/j.jbiotec.2016.02.006.

[11] R.J.I. Knoop, W. Vogelzang, J. van Haveren, D.S. Van Es, High molecular weight poly(ethylene-2,5-furanoate); Critical aspects in synthesis and mechanical property determination, J. Polym. Sci. Part A Polym. Chem. 51 (2013) 4191-4199. doi:10.1002/pola.26833.

[12] J. Zhu, J. Cai, W. Xie, P.H. Chen, M. Gazzano, M. Scandola, R.A. Gross, Poly(butylene 2,5-furan dicarboxylate), a biobased alternative to PBT: Synthesis, physical properties, and crystal structure, Macromolecules. 46 (2013) 796-804. doi:10.1021/ma3023298. 
[13] D.J. Brunelle, J.E. Bradt, J. Serth-Guzzo, T. Takekoshi, T.L. Evans, E.J. Pearce, Semicrystalline polymers via ring-opening polymerization: Prepation and polymerization of alkylene phthalate cyclic oligomers, Macromolecules. 31 (1998) 4782-4790.

[14] Y. Yu, G. Storti, M. Morbidelli, Ring-opening polymerization of L,L-lactide: Kinetic and modeling study, Macromolecules. 42 (2009) 8187-8197. doi:10.1021/ma901359x.

[15] S. Penczek, R. Szymanski, A. Duda, J. Baran, Living Polymerization of Cyclic Esters A Route to (Bio)degradable Polymers. Influence of Chain Transfer to Polymer on Livingness, Macromol. Symp. 201 (2003) 261-269. doi:10.1002/masy.200351129.

[16] M. Liu, N. Vladimirov, J.M.J. Fréchet, A new approach to hyperbranched polymers by ring-opening polymerization of an $\mathrm{AB}$ monomer: 4-(2-Hydroxyethyl)-e-caprolactone, Macromolecules. 32 (1999) 6881-6884. doi:10.1021/ma990785x.

[17] A.-C. Albertsson, I.K. Varma, Recent Developments in Ring Opening Polymerization of Lactones for Biomedical Applications, Biomacromolecules. 4 (2003) 1466-1486. doi:10.1021/bm034247a.

[18] C. Guerrero-Sanchez, R. Hoogenboom, U.S. Schubert, Fast and "green” living cationic ring opening polymerization of 2-ethyl-2-oxazoline in ionic liquids under microwave irradiation, Chem. Commun. (2006) 3797-3799. doi:10.1039/B608364A.

[19] M. Divandari, G. Morgese, L. Trachsel, M. Romio, E.S. Dehghani, J.-G. Rosenboom, C. Paradisi, M. Zenobi-Wong, S.N. Ramakrishna, E.M. Benetti, Topology Effects on the Structural and Physicochemical Properties of Polymer Brushes, Macromolecules. 50 (2017). doi:10.1021/acs.macromol.7b01720.

[20] N.E. Kamber, W. Jeong, R.M. Waymouth, R.C. Pratt, B.G.G. Lohmeijer, J.L. Hedrick, Organocatalytic ring-opening polymerization, Chem. Rev. 107 (2007) 5813-5840. doi:10.1021/cr068415b. 
[21] D. Brunelle, Macrocycles for the synthesis of high molecular weight polymers, in: New Methods Polym. Synth., 1995. http://link.springer.com/chapter/10.1007/978-94-0110607-8_6.

[22] Y. Yu, E.J. Fischer, G. Storti, M. Morbidelli, Modeling of Molecular Weight Distribution in Ring-Opening Polymerization of L,L-Lactide, Ind. Eng. Chem. Res. 53 (2014) 73337342.

[23] S. Penczek, A. Duda, R. Szymanski, Intra- and intermolecular chain transfer to macromolecules with chain scission. The case of cyclic esters, Macromol. Symp. 132 (1998) 441-449. doi:10.1002/masy.19981320141.

[24] H. Chen, W. Yu, C. Zhou, Entropically-Driven Ring-Opening Polymerization of Cyclic Butylene Terephthalate: Rheology and Kinetics, Polym. Eng. Sci. 52 (2012) 91-101. 